doi:10.4149/neo_2015_100

\title{
Immunoparesis in MGUS - Relationship of uninvolved immunoglobulin pair suppression and polyclonal immunoglobuline levels to MGUS risk categories
}

\author{
T. PIKA ${ }^{1, *}$, P. LOCHMAN ${ }^{2}$, V. SANDECKA ${ }^{3}$, V. MAISNAR ${ }^{4}$, J. MINARIK ${ }^{1}$, M. TICHY ${ }^{5}$, J. ZAPLETALOVA ${ }^{6}$, L. SOLCOVA ${ }^{7}$, V. SCUDLA ${ }^{1}$, R. HAJEK ${ }^{8}$
}

${ }^{1}$ Department of Hematooncology, University Hospital Olomouc, Czech Republic; ${ }^{2}$ Department of Clinical Biochemistry, University Hospital Olomouc, Czech Republic; ${ }^{3}$ Department of Internal Medicine and Hematooncology, University Hospital Brno, Czech Republic; ${ }^{4}$ Department of Clinical Hematology 4th Department of Internal Medicine, Charles University Hospital, Hradec Kralove, Czech Republic; ${ }^{5}$ Institute of Clinical Biochemistry and Diagnostics, Charles University Hospital, Hradec Kralove, Czech Republic; ${ }^{6}$ Department of Medical Biophysic, Palacky University Olomouc, Czech Republic; ${ }^{7}$ Department of Clinical Biochemistry, Regional Hospital Trutnov, Czech Republic; ${ }^{8}$ Department of Hematooncology, University Hospital Ostrava, Czech Republic

${ }^{*}$ Correspondence: tomas.pika@seznam.cz

Received August 7, 2014 / Accepted May 27, 2015

\begin{abstract}
Monoclonal gammopathy of undetermined significance (MGUS) is an asymptomatic, potentially malignant condition. It has been established that annually approximately 1-2\% of MGUS cases transforms into one of the malignant forms of monoclonal gammopathies. Progression risk factors include the quantity and type of M-protein, and namely the ratio of free light immunoglobulin chains (FLC). These factors, enable purposeful stratification of MGUS individuals. Some authors consider suppression of polyclonal immunoglobulin levels to be another progression factor. The aim of the study was to compare polyclonal immunoglobulin (PIg) levels with uninvolved heavy/light chain pair (HLC) levels in order to verify the degree of immunoparesis depending on MGUS risk category (0-3). The analyzed set consisted of 159 serum samples from MGUS patients (102 IgG, $57 \mathrm{IgA}$ ), who were stratified into 4 risk groups ( 0 - low, 1 - low-intermediate, 2 - high-intermediate and 3 - high risk of transformation). The results of analysis showed that with increasing degree of MGUS increases risk of immune paresis defined by decreasing levels of polyclonal immunoglobulins, ie. IgA and IgM in the case of IgG MGUS, respectively, $\operatorname{IgG}$ and IgM in case of IgA MGUS. Significant differences were also found when analyzing the levels of uninvolved HLC pairs IgG kappa (resp. IgG lambda) in IgG lambda (IgG kappa) dominant secretion. In the case of MGUS with IgA isotype, the results were similar. Discovery of the connection between the degree of immunosuppression and the level of MGUS risk contributes to our understanding of the relationship between biology, development and potential malignant transformation of MGUS. It is apparent that uninvolved HLC pair assay enables more reliable identification of at-risk MGUS patients than a simple quantitative assay for polyclonal immunoglobulins alone.
\end{abstract}

Key words: MGUS, heavy/light chain, immunoparesis, risk stratification

Monoclonal gammopathy (MG) is a heterogeneous group of diseases characterized by the presence of monoclonal immunoglobulin (MIg) molecules or their fragments (mostly light, but sporadically heavy chains), detectable in the serum and/or urine. Electrophoresis, commonly used for the characterization of serum M-protein, counts among basic examination methods. It enables quantitative assay and immunofixation electrophoresis, a qualitative method for determining the type of M-protein [1]. Free light chain (FLC) serum level assay is currently widely available. It employs nephelometry or turbidimetry along with highly specific detection antibodies against the inner epitopes of free light chains kappa $(\kappa)$ and lambda $(\lambda)$. The examination yields exact quantification of the chains and their mutual $\kappa / \lambda$ ratio - that is, a light chain clonality index which indicates also clonality of the present plasmocyte population $[2,3]$. The newest addition to the spectrum of monoclonal protein assays is the HevyLite ${ }^{\mathrm{TM}}$ system, based on the application of a couple of specific antibodies against junctional epitopes between the domains of heavy and light chain within the constant 
Table 1. Risk stratification system for monoclonal gammopathy of undetermined significance according to Mayo clinic.[18].

\section{Risk factors:}

1. M-protein in serum $<15 \mathrm{~g} / \mathrm{l}$

2. IgG isotype

3. Normal $\kappa / \lambda$ ratio of serum free light chains

Stratification model:

Low risk - normal values of all 3 factors

Low-intermediate risk - abnormity of 1 factor

High-intermediate risk - abnormity of 2 factors

High risk - abnormity of all 3 factors

part of the immunoglobulin chains $[4,5]$. Depending on the type of the kit used, the method permits HLC level assay of various immunoglobulin isotypes (IgG, $\operatorname{IgA}, \operatorname{IgM})$ and yields quantitative results even in cases where electrophoretic quantitative assay of M-protein is not possible. Calculation of the HLC index - Igא/Ig $\lambda$ clonality index is possible, in a manner analogous to the free light chain serum level assay.

Monoclonal gammopathy of undetermined significance (MGUS) denotes an asymptomatic, potentially malignant condition characterized by benign clonal proliferation of plasma cells secreting MIg detectable in serum and/or urine, in the absence of lymphoproliferative malignancies $[1,6]$. MGUS accounts for about $60 \%$ of all monoclonal gammopathies, and $1-2 \%$ of MGUS cases annually transforms into one of the malignant MGs [7]. A number of studies targeted identification of risk factors determining the risk level and probability of MGUS transformation into one of the malignant MG forms. The MIg concentration and isotype were identified as significant among serological methods. The presence of BenceJones protein in the urine and the suppression of polyclonal immunoglobulins is considered another significant predictor by some of the authors [ $8-11]$. The free light chain serum level assay along with the $\kappa / \lambda$ clonality index calculation enabled more precise identification of individuals with higher risk of transformation into malignant MG forms. A combination of the $\kappa / \lambda$ clonality index with the M-protein quantity and isotype was used in the generally accepted Mayo clinic stratification system, which divides the patients into 4 prognostically specific groups with recommendations for optimal monitoring (Table 1) [12 - 14].

The objective of this study was an assessment of the degree of immunoparesis, defined by polyclonal immunoglobulin level suppression and the degree of suppression of the uninvolved immunoglobulin heavy-light pair, for individual stages of MGUS risk stratification in the IgG and IgA MGUS sets. Turbidimetry system SPA Plus (The Binding Site) was used for the laboratory analyses.

\section{Patients and methods}

Patients. Frozen serum samples from serum banks of University Hospitals in Olomouc, Brno Bohunice and Hradec Kralove, with known isotype and M-component quantity, determined by standard analytic methods, were used for the analysis. The analyzed set comprised 102 individuals with IgG MGUS (secretion ratio: kappa 52\% $(\mathrm{n}=53) /$ lambda $48 \%(\mathrm{n}=49))$. According to Mayo clinic stratification system, $32 \%(n=33)$ of the individuals met the low risk criteria, $46 \%(\mathrm{n}=47)$ low-intermediate risk criteria, and 22\% ( $n=22)$ high-intermediate risk criteria. The analyzed set of the IgA isotype MGUS comprised 57 individuals (secretion ratio: kappa 51\% $(\mathrm{n}=29) /$ lambda $49 \%(n=28)$, where $44 \%(n=25)$ were in the low-intermediate, $37 \%(\mathrm{n}=21)$ in the high-intermediate, and $19 \%(\mathrm{n}=11)$ in the high risk group for malignant transformation.

Methods. Free light chain serum level assays were carried out using the FreeLite SPA Plus kappa free kit (normal range (NR): 33 - 19,4 mg/l) and FreeLite SPA Plus lambda free kit (NR: $5,71-26,3 \mathrm{mg} / \mathrm{l}$ ) with subsequent clonality index calculation. Polyclonal immunoglobulin levels were determined with the Human IgG kit (NR: 7 - 16 g/l), Human IgA kit (0,7 - 4 $\mathrm{g} / \mathrm{l})$ and Human IgM kit for the SPA Plus $(0,4-2,3 \mathrm{~g} / \mathrm{l})$. HLC levels were determined using the HevyLite Human IgG kappa (NR: 3,84-12,07 g/l), IgG lambda (NR: 1,91 - 6,74 g/l), IgA kappa (NR: 0,57 - 2,08 g/l) and IgA lambda (NR: 0,44 - 2,04 $\mathrm{g} / \mathrm{l})$ kits for the SPA Plus. The ratio of the immunoglobulin isotype pairs was calculated. The assays were performed with a SPA Plus turbidimeter (The Binding Site) at the Department of Clinical Biochemistry, University Hospital Olomouc, in order to avoid potential inter-laboratory errors. Some of the samples had to be manually pre-diluted, because of extreme concentrations outside the turbidimeter range. Statistical analysis was performed by the Mann-Whitney $U$ test with Bonferroni adjustment.

\section{Results}

Comparison of the degree of suppression of polyclonal immunoglobulin levels in the individual risk groups of the

Table 2. Suppression of polyclonal immunoglobulin and uninvolved isotype HLC pair levels in each risk group of IgG monoclonal gammopathy of undetermined significance.

\begin{tabular}{lccc}
\hline MGUS Risk: & Suppression of polyclonal IgA & Suppression of polyclonal IgM & Suppression of uninvolved IgG HLC pair \\
\hline Low $(\mathrm{n}=33)$ & $6 \%(2 / 33)$ & $9 \%(3 / 33)$ & $39 \%(13 / 33)$ \\
Low-intermediate $(\mathrm{n}=47)$ & $25 \%(12 / 47)$ & $10 \%(5 / 47)$ & $45 \%(21 / 47)$ \\
High-intermediate $(\mathrm{n}=22)$ & $45 \%(10 / 22)$ & $41 \%(9 / 22)$ & $100 \%(22 / 22)$ \\
\hline
\end{tabular}




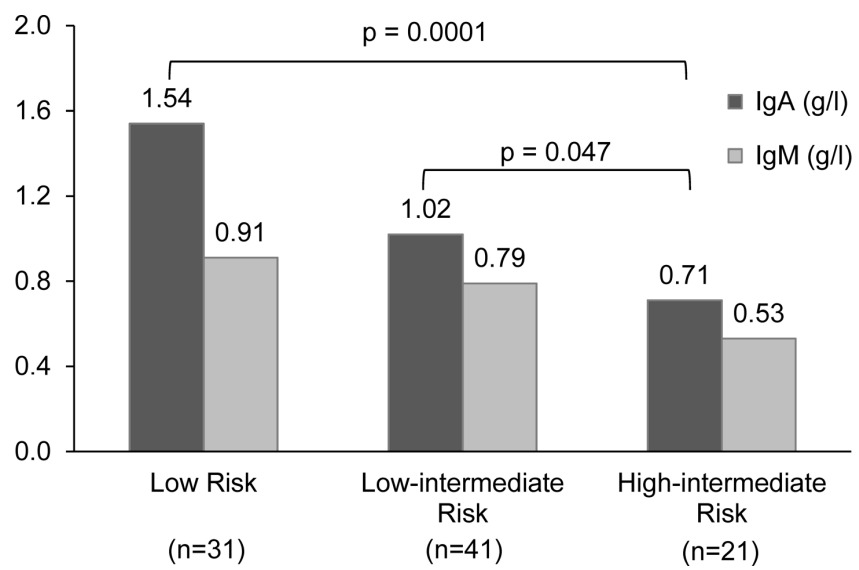

Figure 1. The comparison of polyclonal IgA and IgM immunoglobulin levels between risk categories in IgG MGUS.

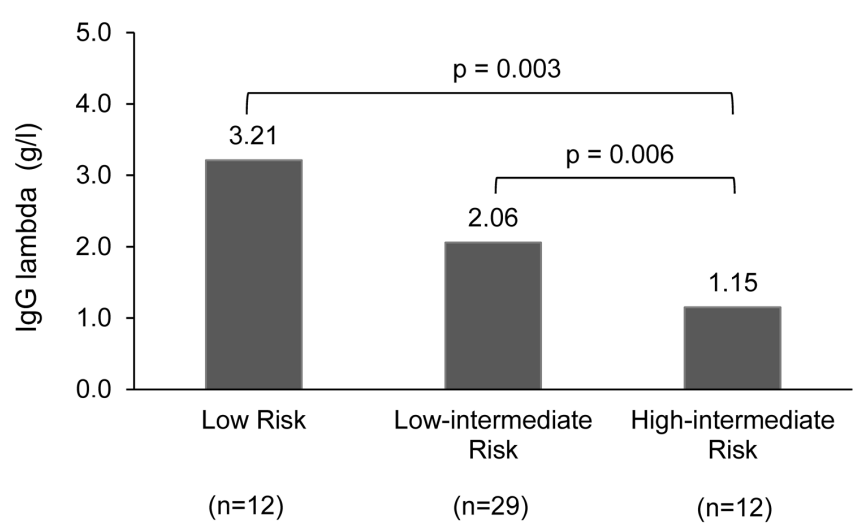

Figure 2. The comparison of uninvolved IgG lambda levels determined by HevyLite $^{\mathrm{TM}}$ between risk categories in IgG kappa MGUS.

IgG MGUS set revealed significantly higher immunoglobulin IgA levels in patients with low or low-intermediate risk compared to the high-intermediate risk group $(\mathrm{p}=0,0001$ and $\mathrm{p}=0,047$, respectively) (Fig. 1, Table 2). The groups did not differ significantly in the levels of immunoglobulin IgM, but the trend of levels decreasing with the degree of MGUS risk was preserved, too. Comparison of uninvolved isotype pairs between individual risk groups in individuals with dominant IgGK secretion showed significantly higher levels of the uninvolved IgG $\lambda$ pair in the low and low-intermediate groups

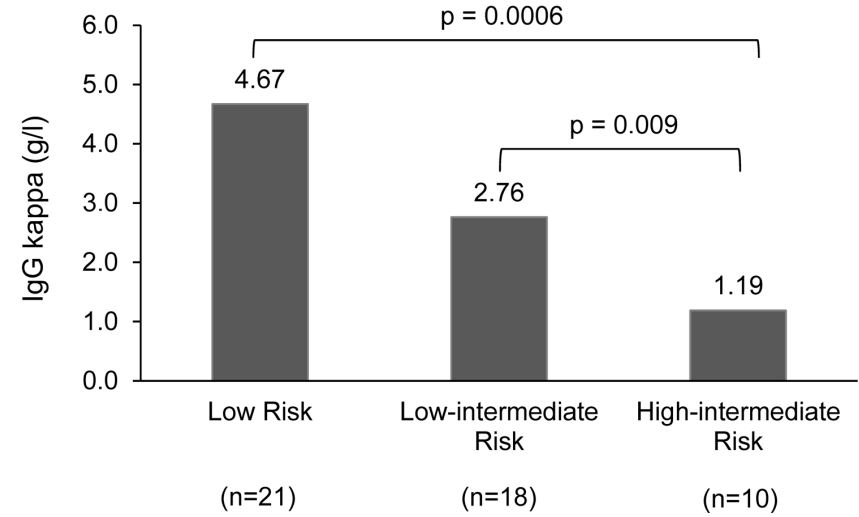

Figure 3. The comparison of uninvolved IgG kappa levels determined by HevyLite $^{\mathrm{TM}}$ between risk categories in IgG lambda MGUS.

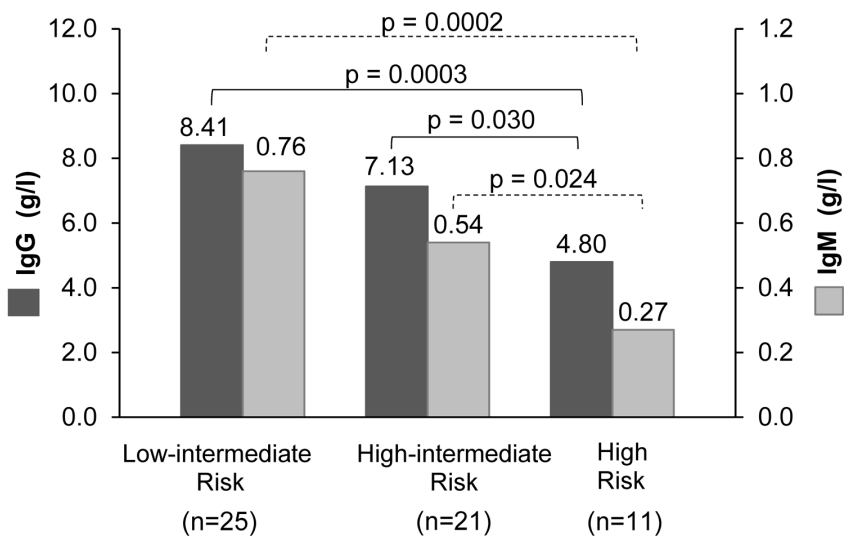

Figure 4. The comparison of polyclonal IgG and IgM immunoglobulin levels between risk categories in IgA MGUS.

than in the high-intermediate risk group $(\mathrm{p}=0,003$ and $\mathrm{p}=$ 0,006 , respectively) (Fig. 2). In case of dominant IgG $\lambda$ secretion, significantly higher IgGk levels were found in individuals from the low and low-intermediate risk groups than from the high-intermediate risk group $(\mathrm{p}=0,0006$ and $\mathrm{p}=0,009$, respectively) (Fig. 3).

Analysis of the MGUS group with IgA isotype revealed significantly higher immunoglobulin IgG levels in the groups with low-intermediate and high-intermediate risk than in the high risk group $(\mathrm{p}=0,0003$ and $\mathrm{p}=0,03$, respectively)

Table 3. Suppression of polyclonal immunoglobulin and uninvolved isotype HLC pair levels in each risk group of IgA monoclonal gammopathy of undetermined significance.

\begin{tabular}{lccc}
\hline MGUS Risk: & Suppression of polyclonal IgG & Suppression of polyclonal IgM & Suppression of uninvolved IgA HLC pair \\
\hline Low-intermediate $(\mathrm{n}=25)$ & $28 \%(7 / 25)$ & $12 \%(3 / 25)$ & $16 \%(4 / 25)$ \\
High-intermediate $(\mathrm{n}=21)$ & $43 \%(9 / 21)$ & $33 \%(7 / 21)$ & $47 \%(10 / 21)$ \\
High $(\mathrm{n}=11)$ & $82 \%(9 / 11)$ & $82 \%(9 / 11)$ & $91 \%(10 / 11)$ \\
\hline
\end{tabular}




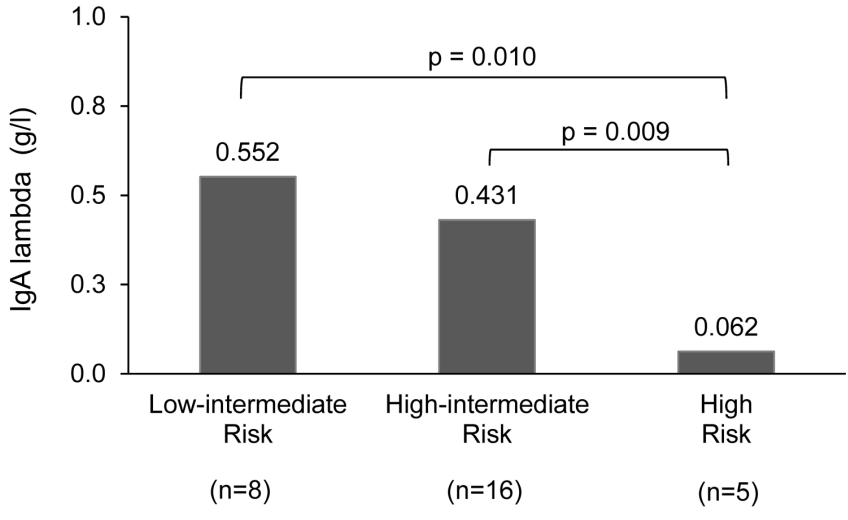

Figure 5. The comparison of uninvolved IgA lambda levels determined by HevyLite $^{\mathrm{TM}}$ between risk categories in IgA kappa MGUS.

(Fig.4, Table 3). IgM levels were likewise significantly higher in the groups with low-intermediate and high-intermediate risk than in the high-risk group $(\mathrm{p}=0,0002$ and $\mathrm{p}=0,024$, respectively).

Comparison of the uninvolved isotype pairs in individuals

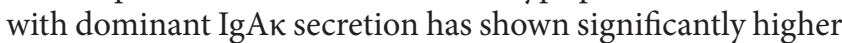
levels of the uninvolved $\operatorname{Ig} A \lambda$ pair in the low-intermediate and high-intermediate groups than in the high-intermediate risk group ( $p=0,01$ and $p=0,009$, respectively) (Fig. 5). In case of dominant $\operatorname{Ig} A \lambda$ secretion, significantly higher $\operatorname{Ig} A \kappa$ levels were found in the low-intermediate risk individuals than in the high risk group ( $\mathrm{p}=0,005)$ (Fig. 6).

\section{Discussion}

Discovery of transformation risk factors with the prospect of identification and early intervention for MGUS patients with high risk of malignant monoclonal gammopathy development is the scientific objective of many research teams. Very promising are modern flow cytometry methods with aberrant phenotype identification, molecular - cytogenetic methods or gene expression analyse. Still, the main indicators are the commonly available risk factor indicators such as $\mathrm{M}$-protein type and its quantity. Some publications mention also the presence of Bence-Jones protein in the urine, high lactate dehydrogenase activity and, the FLC serum level assay [12, 15 - 17]. An extensive analysis of 1148 MGUS individuals related to transformation risk factors was published by Rajkumar [12]. In addition to more broadly accepted parameters - quantity and isotype of the M-protein (IgA, IgM, biclonal $\operatorname{Ig} \mathrm{A}+\operatorname{IgM}$ ) - the analysis of absolute FLC serum levels revealed that patients with elevated FLCs are at far higher risk of transformation into one of the malignant $\mathrm{MG}$ forms than patients with low FLC levels. Moreover, multivariate analysis has shown that the values of $\kappa / \lambda$ index are more convincing, as the individuals with abnormal value of this index presented higher transformation risk than individuals whose $\kappa / \lambda$ index

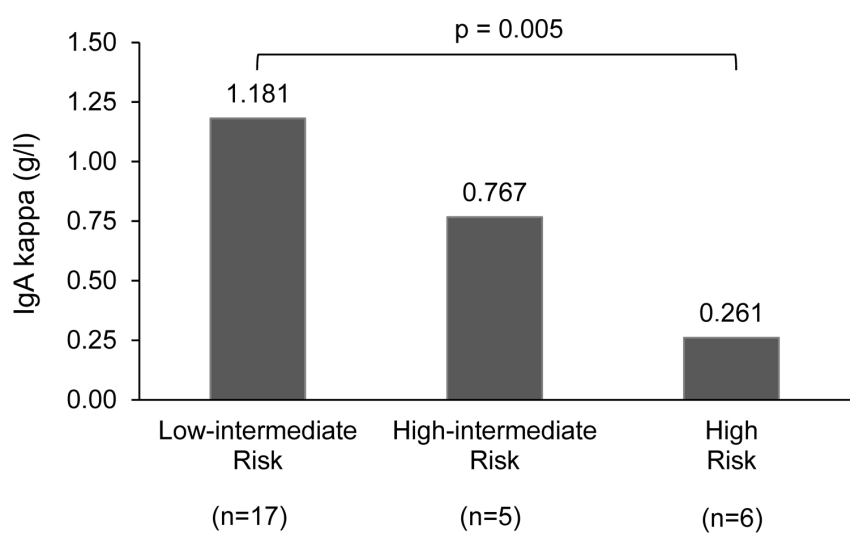

Figure 6. The comparison of uninvolved $\operatorname{IgA}$ kappa levels determined by HevyLite $^{\mathrm{TM}}$ between risk categories in IgA lambda MGUS.

was within physiological limits. This indicator was independent on other evaluated parameters. By combining the $\kappa / \lambda$ index value, $M$-protein quantity and $M$-protein isotype as selection criteria, the authors have created a stratification system capable of quite reliable identification of individuals with higher risk of transformation. The stratification system was widely accepted and incorporated into the International Myeloma Working Group (IMWG) recommendations for MGUS [13]. The presence of suppressed polyclonal immunoglobulin levels is often mentioned as another MGUS progression risk factor. It used to be one of the "minor" criteria of the Multiple Myeloma Diagnostic System of the Southwest Oncology Group (SWOG). It has been established that a developed multiple myeloma is associated with suppressed polyclonal immunoglobulin levels of the uninvolved classes [18]. Similarly, Bence-Jones or nonsecretory myeloma form is associated with suppression of all polyclonal immunoglobulin classes. Our study focused on a concomitant quantitative evaluation of PIg suppression and uninvolved HLC pair level suppression between MGUS risk categories, as it appears that publications analyzing relationship of these parameters are lacking.

Immunoparesis, occuring frequently in developed multiple myeloma, is associated with a decrease in functional B-cells and later also T-cells. The polyclonal immunoglobulin level assay, which reveals production rate of the "non-neoplastic" plasmocytes, is the best identification method for immunoparesis. Some authors consider polyclonal immunoglobulin suppression one of the risk factors of MGUS transformation into MM $[8,9]$. We have confirmed the relation of this immunoparesis to the MGUS risk levels in our analysis set. Individuals with high transformation risk have not only polyclonal immunoglobulin levels frequently below the physiological limit, but the difference is also significant in absolute levels. This phenomenon is associated with an inhibition of normal immunocompetent cells as a result of prominent expansion of the neoplastic clone. It can be expected that representation of the neoplastic clone increases within the 
individual risk categories (0-3), along with inhibition of the alternative normal $\mathrm{B}$-cell clone and the level of the reduction of the uninvolved HLC pair it produces. Evaluation of residual production of the "non-neoplastic" immunoglobulin, which is of the same type as the M-protein produced by neoplastic plasmocytes, was not possible on principle, because a nephelometric or turbidimetric assay registers of the total sum of polyclonal and monoclonal immunoglobulins. To-date, an uninvolved immunoglobulin assay has been available, that is a quantitative assay of light $\kappa$ and $\lambda$ immunoglobulin chain with subsequent calculation of the $\kappa / \lambda$ index, where low levels of the uninvolved chain to a certain degree indicated the state of residual "non-neoplastic" secretion. The uninvolved HLC pair assay allows to evaluate the residual secretion of the "non-neoplastic" plasmocyte population producing an immunoglobulin with heavy chain identical to the M-protein type, but with a different light chain. Our study confirmed the close relationship of the degree of immunoparesis defined by polyclonal immunoglobulin levels and the degree of uninvolved HLC pair level suppression to the risk of MGUS transformation. The results of both types of the assay are comparable. In the case of the IgG type, the differences in the level of the alternative pair are more pronounced, which is in concordance with the conclusions of Katzmann et al., who described in a study aimed at MGUS individuals significant suppression of the alternative HLC pair in the IgG class more pronounced than in other classes. The main contribution of this study is, however, the definitive demonstration of prognostic importance of the alternative HLC pair suppression. Adding this parameter to Mayo clinic stratification system allows for a better stratification of MGUS individuals, and particularly for easier identification and monitoring of patients with high risk of transformation into MM [19]. Presented work confirmed our introductory hypothesis about increasing, measurable suppression of PIg levels, but particularly about suppression of uninvolved HLC levels, in relation to risk of MGUS transformation. The presented observation is in agreement with the widely accepted hypothesis about the transformation of MGUS into MM. The results of our analysis have documented an increasing degree of immunoparesis with increasing risk of transformation, and also provided a biological substantiation of the accepted "sub-classification" of MGUS.

\section{Conclusion}

The established association of the degree of immunosuppression with the level of MGUS risk, and particularly the obvious contribution of the uninvolved HLC pair assay, add another aspect to the understanding of the biology, development and potential malignant transformation of MGUS with the advantage of another measurable parameter. HLC assay, the uninvolved HLC pair assay in particular, enables improved identification of individuals with higher risk of transformation into a malignant type of monoclonal gammopathy.
Acknowledgments: This work was supported by the grants IGA MZ CR NT12451/5, NT14400, NT12215 and by the company The Binding Site s.r.o., Czech Republic.

\section{References}

[1] The International Myeloma Working Group. Criteria for the classification of monoclonal gammopathies, multiple myeloma and related disorders: a report of the International Myeloma Working Group. Brit J Haematol 2003; 121: 749757. http://dx.doi.org/10.1046/j.1365-2141.2003.04355.x

[2] BRADWELL AR. Serum free light chain measurements move to center stage. Clin Chem 2005, 51, 5, p. 805-807. http:// dx.doi.org/10.1373/clinchem.2005.048017

[3] BRADWELL AR, CARR-SMITH HD, MEAD GP, TANG LX, SHOWELL PJ et al. Highly sensitive, automated immunoassay for immunoglobulin free light chains in serum and urine. Clin Chem 2001; 47: 673-680.

[4] BRADWELL AR. Analysis of immunoglobulin heavy chain/ light chain pairs (HevyliteTM). In: Bradwell AR. Serum free light chain analysis, 6th edition. Birmingham, UK, The Binding Site Ltd.; 2010: 301-320.

[5] BRADWELL AR, HARDING S, FOURRIER NJ, WALLIS GL, DRAYSON MT et al. Assesment of monoclonal gammopathies by nephelometric measurement of individual immunoglobulin kappa/lambda ratios. Clin Chem 2009, 55: 1646-1655. http://dx.doi.org/10.1373/ clinchem.2009.123828

[6] KYLE RA, THERNEAU TM, RAJKUMAR SV, LARSON DR, PLEVAK MF et al. Prevalence of monoclonal gammopathy of undetermined significance. N Engl J Med 2006; 354: 1362-1369. http://dx.doi.org/10.1056/NEJMoa054494

[7] KYLE RA, THERNEAU TM, RAJKUMAR SV, OFFORD JR, LARSON DR et al. A long-term study of prognosis in monoclonal gammopathy of undetermined significance. $\mathrm{N}$ Engl J Med 2002; 346: 564-569. http://dx.doi.org/10.1056/ NEJMoa01133202

[8] BALDINI L, GUFFANTI A, CESANA BM, COLOMBI M, CHIORBOLI O et al. Role of different hematologic variables in defining the risk of malignant transformation in monoclonal gammopathy. Blood 1996; 87: 912-918.

[9] CESANA C, KLERSY C, BARBARANO L, NOSARI AM, CRUGNOLA $\mathrm{M}$ et al. Prognostic factors for malignant transformation in monoclonal gammopathy of undetermined significance and smoldering multiple myeloma. J Clin Oncol 2002; 20: 1625-1634. http://dx.doi.org/10.1200/ JCO.20.6.1625

[10] VENERI D, AGEL H, FRANCHINI M, KRAMPERA M, ZANOTTI $R$ et al. Malignant evolution of monoclonal gammopathy of undetermined significance: analysis of 633 consecutive cases with a long term follow-up. Haematologica 2004; 89: 876-878.

[11] SACKMANN F, PAVLOVSKY MA, CORRADO C, PIZZOLATO M, ALEJANDRE $M$ et al. Prognostic factors in monoclonal gammopathy of undetermined significance. Haematologica 2008; 93: 153-154. http://dx.doi.org/10.3324/ haematol.11744 
[12] RAJKUMAR SV, KYLE RA, THERNEAU TM, MELTON LJ, BRADWELL AR et al. Serum free light chain ratio is an independent risk factor for progression in monoclonal gammopathy of undetermined significance. Blood 2005; 106: 812-817. http://dx.doi.org/10.1182/blood-2005-03-1038

[13] KYLE RA, DURIE BGM, LANDGREN O, LANDGREN O, BLADE $J$ et al. Monoclonal gammopathy of undetermined significance (MGUS) and smoldering (asymptomatic) multiple myeloma: IMWG consensus perspectives risk factors for progression and guidelines for monitoring and management. Leukemia 2010; 24: 1121-1127. http://dx.doi.org/10.1038/leu.2010.60

[14] BIANCHI G, KYLE RA, COLBY CL, LARSON DR, KUMAR S et al. Impact of optimal follow-up of monoclonal gammopathy of undetermined significance (MGUS) on early diagnosis and prevention of myeloma-related complications. Blood 2010; 116: 2019-2025. http://dx.doi.org/10.1182/blood-2010-04-277566

[15] MAILANKODY S, MENA E, YUAN CM, BALAKUMARAN A, KUEHL WM et al. Molecular and biologic markers of progression in monoclonal gammopathy of undetermined significance to multiple myeloma. Leuk Lymph 2010; 51:2159-2170. http:// dx.doi.org/10.3109/10428194.2010.525725
[16] PEREZ-PERSONA E, VIDRIALES MB, MATEO G, GARCIASANZ R, MATEOS MV et al. New criteria to identify risk of progression in monoclonal gammopathy of uncertain significance and smoldering multiple myeloma based on multiparameter flow cytometry analysis of bone marrow plasma cells. Blood 2007; 110: 2586-2592. http://dx.doi.org/10.1182/ blood-2007-05-088443

[17] KOVAROVA L, BURESOVA I, BUCHLER T, SUSKA R, POUR L et al. Phenotype of plasma cells in multiple myeloma and monoclonal gammopathy of undetermined significance. Neoplasma 2009; 56. 526-532. http://dx.doi.org/10.4149/ neo $2009 \quad 06 \quad 526$

[18] DURIE BGM, SALMON SE. Multiple myeloma, macroglobulinemia and monoclonal gammopathies. In: Hoffbrand AV, Blain MC, Hirsh J. Recent advances in hematology. Churchill Livingstone, New York, 1977: 243-261.

[19] KATZMANN JA, CLARK R, KYLE RA, LARSON DR, THERNEAU TM et al. Suppresion of uninvolved immunoglobulins defined by heavy/light chain pair suppression is risk factor for progression of MGUS. Leukemia 2012; 27: 208-212. http://dx.doi.org/10.1038/leu.2012.189 